\title{
PLANTEAMIENTO TERAPÉUTICO DE LAS FÍSTULAS VESICOGENITALES. ANÁLISIS RETROSPECTIVO DE NUESTRA SERIE
}

\author{
F.J. NAVARRO SEBASTIÁN, J.I. GARCÍA GONZÁLEZ, M. CASTRO PITA, \\ J.M. DIEZ RODRÍGUEZ, M. ARRIZABALAGA MORENO, A. MAÑAS PELILLO, \\ P. PANIAGUA ANDRÉS.
}

Servicio de Urología. Hospital de Móstoles. Madrid.

Actas Urol Esp. 27 (7): 530-537, 2003

\section{RESUMEN}

PLANTEAMIENTO TERAPÉUTICO DE LAS FÍSTULAS VESICOGENITALES. ANÁLISIS RETROSPECTIVO DE NUESTRA SERIE

Las fístulas vesicogenitales son comunicaciones anómalas de la vejiga urinaria con cualquier parte del aparato genital femenino. En el presente trabajo analizamos nuestra serie de fístulas vesicogenitales diagnosticadas y tratadas desde 1986, analizando las causas etiológicas, tratamientos aplicados, complicaciones y resultados de los mismos.

El número total de fístulas han sido 20 (18 vesicovaginales y 2 vesicouterinas). De las fístulas vesicovaginales $15(83 \%)$ eran yatrógenas y $3(17 \%)$ neoplásicas. Las fístulas vesicouterinas aparecieron tras sendas cesáreas.

El tratamiento inicial de las fístulas yatrógenas fue conservador con sondaje vesical, produciéndose su resolución en el 20\% de las mismas (3 casos). En el resto de las pacientes se realizó tratamiento quirúrgico por vía abdominal con distintas técnicas según el tipo de fístula, intensidad y edad de la paciente, resultando satisfactorio en el 91\% de los casos. La utilización de técnicas sencillas que generan menor morbilidad así como la actuación precoz no implica un peor resultado en las fístulas yatrógenas simples.

PALABRAS CLAVE: Fístula vesical. Fístula vesicovaginal. Fístula vesicouterina. Incontinencia urinaria.

\section{ABSTRACT}

TREATMENT APPROACH FOR VESICOGENITAL FISTULA. RESTROSPECTIVE ANALYSIS OF OUR DATA

The vesicogenital fistula are abnormal communications between female genitalia and urinary bladder. We recorded all the vesicogenital fistula diagnosed since 1986, analyzing aetiology, treatment applied, complications and results.

Total number of fistula have been 20 (18 vesicovaginal and 2 vesicouterine). The distribution in vesicovaginal fistula was iatrogenic in 15 cases $(83 \%)$ and tumoral in 3 cases (17\%). Vesicouterine fistula were due to cesarea.

The initial treatment of the iatrogenic fistula was conservative using foley catheter. Twenty percent of the patients were cured with this treatment (3 cases). Surgical repair was necessary for the other patients, using different surgical approach according to the type of the fistula, intensity and patient age. It was successful in $91 \%$ of the patients. The results shows that simple surgical approach generate less morbidity and the early intervention is not less efficient.

KEY WORDS: Bladder fistula. Vesicovaginal fistula. Vesicouterine fistula. Urinary incontinence. 
$\mathrm{L}^{2}$ as fístulas vesicogenitales son comunicaciones anómalas de la vejiga urinaria con cualquier parte del aparato genital femenino. Fundamentalmente son de dos tipos: vesicovaginales (FVV) y vesicouterinas (FVU). No obstante, excepcionalmente pueden presentarse también fístulas vesicoanexiales (ováricas y tubáricas) ${ }^{1-3}$.

Se ha descrito algún caso aislado de fístula vesicogenital congénita, aunque la gran mayoría son adquiridas ${ }^{4}$

Actualmente la causa más frecuente de fístula vesicovaginal, en los países desarrollados, es la lesión yatrógena, sobre todo tras cirugía ginecológica $\mathrm{u}$ obstétrica y ocasionalmente tras cirugía urológica o intestinal. Representan alrededor del 80\% de las mismas. La incidencia de lesión yatrógena tras intervención ginecológica varía entre 0,36-2 \%, según distintos autores ${ }^{5-7}$. Mientras tanto, la causa más frecuente en los países subdesarrollados sigue siendo la obstétrica, con una incidencia que va desde el 65 al 96\% $\%^{8-10}$.

Las fístulas vesicouterinas, mucho menos frecuentes que las vesicovaginales, se producen fundamentalmente como consecuencia de cesáreas.

Sólo en un pequeño porcentaje de casos conseguiremos la resolución de estas fístulas con medidas conservadoras (sonda vesical, hormonoterapia, etc.). En los restantes será preciso realizar un tratamiento quirúrgico.

Presentamos nuestra serie de fístulas vesicogenitales, analizando las causas etiológicas, tratamientos aplicados, complicaciones y resultado de los mismos.

\section{MATERIAL Y MÉTODOS}

Hemos realizado un estudio retrospectivo en el periodo comprendido entre enero de 1986 y diciembre de 2001. Han sido diagnosticadas en nuestro centro 20 fístulas vesicogenitales: 18 vesicovaginales (90\%) y 2 vesicouterinas (10\%). Dentro de las fístulas vesicovaginales la mayoría eran secundarias a yatrogenia ginecológica $u$ obstétrica 15 (83\%) y el resto eran neoplásicas 3 (17\%) ( 2 tras recidiva de carcinoma de cérvix uterino, 1 tras recidiva de carcinoma endometrial).

En los últimos 10 años, han sido practicadas en nuestro hospital, por parte del servicio de ginecología, 2.291 histerectomías, habiéndose producido en ese periodo de tiempo 10 fístulas vesicovaginales, lo que representa una incidencia del 0,44\%. Respecto a la histología de los casos fistulizados, en 9 ocasiones se trataba de patología benigna y en 1 maligna.

El diagnóstico de las mismas se fundamentó básicamente en tres estudios: exploración genital con espéculo y prueba del colorante, urografía intravenosa y cistovaginoscopia, dejando la cistografía para la comprobación de persistencia o ausencia de fístula tras el mantenimiento de la sonda vesical. En los casos de sospecha de fístula neoplásica se asoció TAC abdominopélvico y biopsia vesical perifistulosa preoperatoria.

Los 15 casos de fístulas yatrógenas tienen una edad media de 44 años (31-46), de ellas 13 (86\%) se produjeron tras histerectomía abdominal, 1 (7\%) tras cesárea y 1 (7\%) tras parto instrumentado. Ninguna había sido previamente operada, considerándolas como fístulas simples en 12 casos y complejas en 3 (2 eran múltiples y 1 estaba radiada).

En todos estos casos se intentó tratamiento conservador (sonda vesical y antibioterapia), con tiempo medio de permanencia del catéter de 3 meses (1-6). Las pacientes en las que fracasó este tratamiento fueron sometidas a cirugía por vía transabdominal. Habitualmente hemos utilizado la técnica de $\mathrm{O}^{\prime}$ Conor, vía transvesical e intraperitoneal, realizando una bipartición vesical, fistulorrafia en varios planos e interposición de un parche peritoneal o de epiplón. Solamente en 3 ocasiones realizamos una fistulectomía y fistulorrafia en varios planos, vía transvesical y extraperitoneal, por tratarse de fístulas simples y de pequeño tamaño.

Los 3 casos de fîstulas neoplásicas tienen una edad media de 62 años $(46,67,74), 2$ de ellas eran secundarias a recidiva de carcinoma de cervix uterino, que habian sido tratadas previamente, una mediante histerectomía y otra con radioterapia. La tercera era secundaria a recidiva de carcinoma de endometrio tratado mediante histerectomía + radioterapia. Se realizaron en 2 ocasiones derivación urinaria alta paliativa (transureterostomía cutánea y nefrostomía percutánea permanente), en el tercer caso correspondiente al carcinoma de cérvix tratado con radioterapia, se practicó una exenteración pélvica anterior, aso- 
ciada a nefrectomía derecha por riñón anulado, resección de íleon terminal por infiltración del mismo y ureterostomía cutánea izquierda.

Las 2 fístulas vesicouterinas, se produjeron tras sendas cesáreas. Se manifestaron clínicamente con incontinencia urinaria en uno de los casos y en el otro con menuria e hipomenorrea (Síndrome de Youseff). Ambas se intervinieron mediante bipartición vesical, fistulorrafia e interponiendo epiplón en un caso y peritoneo en otro.

\section{RESULTADOS}

El tratamiento conservador ha resuelto el 20\% de las fístulas (3/15), con un tiempo medio de permanencia de la sonda de 3 meses (rango 1-6). Se objetivaron datos clínicos de probable curación de la fístula al mes de llevar la sonda, aunque se mantuvo la misma hasta la comprobación cistográfica de dicha resolución. Estas fístulas eran de pequeño tamaño $<0,5 \mathrm{~cm}$ ) y bajo débito, sin presentar recidiva tras un seguimiento medio de 26 meses (rango 25-27).

Las 12 restantes pacientes fueron sometidas a tratamiento quirúrgico. En 2 ocasiones el tratamiento fue precoz (antes de 3 meses) y en 10 fue tardío (Tabla I). En los 3 casos en que se realizó fistulectomía + fistulorrafia por planos, se consiguió la resolución de las mismas en todos los casos. Se trataban de fístulas simples y menores de $1 \mathrm{~cm}$, con buena elasticidad vesical. En 9 ocasiones se realizó bipartición vesical y fistulorrafia por planos (según técnica de O־Conor) interponiendo peritoneo en 6 ocasiones y epiplón en 3 .

\section{TABLA I}

TRATAMIENTO QUIRÚRGICO DE FÍSTULAS VESICOVAGINALES YATRÓGENAS

\begin{tabular}{|l|c|c|c|c|c|}
\hline $\begin{array}{l}\text { Cirugía } \\
\text { abdominal }\end{array}$ & № & Precoz & Tardía & Compleja & $\begin{array}{c}\% \text { de } \\
\text { éxito }\end{array}$ \\
\hline $\begin{array}{l}\text { Fistulectomia }+ \\
\text { Fistulorrafia }\end{array}$ & 3 & 1 & 2 & 0 & 100 \\
\hline $\begin{array}{l}\text { Bipartición + } \\
\text { Epiplón }\end{array}$ & 3 & 0 & 3 & 1 & 100 \\
\hline $\begin{array}{l}\text { Bipartición }+ \\
\text { Peritoneo }\end{array}$ & 6 & 1 & $5 *$ & 2 & $83^{*}$ \\
\hline $\begin{array}{l}\text { Total } \\
\text { Reoperación } \\
* \text { Fistulorrafia }\end{array}$ & 12 & 2 & 10 & 3 & 91,6 \\
\hline
\end{tabular}

Se trataban de 6 fístulas mayores de $1 \mathrm{~cm}, 2$ fístulas con varios trayectos y 1 fístula radiada. Las interposición de epiplón resolvió las fístulas en todos los casos, mientras que la interposición de peritoneo lo consiguió en el 83\% (5/6). El caso fracasado precisó una segunda intervención, objetivándose una fístula de escaso calibre, que se resolvió mediante fistulorrafia por planos.

El éxito global de la serie con la primera intervención es del $91,6 \%$ y del $88,5 \%$ si hacemos referencia a las cirugías con interposición de tejidos, con un seguimiento medio de 34 meses (rango 13-60).

Se presentaron complicaciones post-operatorias en 4 pacientes $(31 \%)$, todas aparecieron en pacientes sometidas a cirugías intraperitoneales y en la reintervenida (Tabla II). Las complicaciones han sido infectivas en 3 casos (shock séptico grave, infección importante de herida quirúrgica con posterior eventración y cuadro de pielonefritis asociado a derrame pleural ipsilateral) y cuadro de hemorragia postoperatoria causante de anemia severa sintomática que precisó varias transfusiones sanguíneas. Se objetivó reflujo vesicoureteral en el primer control cistográfico en 3 pacientes, que había desaparecido a los 6 meses.

De las fístulas por recidiva neoplásica, en dos de ellas se practicó una derivación alta paliativa, teniendo una supervivencia de 6 meses y 2 años

\section{TABLA II}

COMPLICACIONES POST-OPERATORIAS EN LAS FÍSTULAS VESICOVAGINALES YATRÓGENAS

\begin{tabular}{|c|c|c|c|c|c|}
\hline & $\begin{array}{l}\mathrm{F} \\
3\end{array}$ & $\begin{array}{c}\mathrm{B} / \mathrm{E} \\
3\end{array}$ & $\begin{array}{c}\mathrm{B} / \mathrm{P} \\
6\end{array}$ & $\begin{array}{c}\mathrm{R} / \mathrm{F} \\
1\end{array}$ & $\begin{array}{c}\text { Total } \\
13\end{array}$ \\
\hline Schock séptico & - & 1 & & & 1 \\
\hline $\begin{array}{l}\text { Pielonefritis }+ \\
\text { derrame pleural }\end{array}$ & - & 1 & & & 1 \\
\hline $\begin{array}{l}\text { Infec. herida + } \\
\text { eventración }\end{array}$ & - & & & 1 & 1 \\
\hline Anemia severa & - & & 1 & & 1 \\
\hline Total & 0 & 2 & 1 & 1 & $\begin{array}{c}4 \\
31 \%\end{array}$ \\
\hline Reflujo temporal & 1 & 0 & 2 & 0 & 3 \\
\hline
\end{tabular}

F: Fistulorrafia. B/E: Bipartición + epiplón.

B/P: Bipartición + peritoneo. R/F: Reoperación/fistulorrafia. 
respectivamente. La tercera sometida a exenteración pélvica anterior, permanece viva tras un seguimiento de 18 meses.

El tratamiento de las 2 fístulas vesicouterinas consiguió la curación de ambas, sin complicaciones postoperatorias.

\section{DISCUSIÓN}

Las fistulas vesicovaginales, que representan aproximadamente el $90 \%$ de las fístulas vesicogenitales, son conocidas desde tiempos antiguos. Las primeras descripciones datan de hace 2.000 años y corresponden a escritos hindúes ${ }^{6}$. Posteriormente es Avicena en su tratado AlKanoon (hace más de 1.000 años), quien realiza una descripción más precisa indicando su origen en partos prolongados y difíciles. Por otro lado, Derry cita la existencia de una FVV en la momia de una mujer perteneciente a la $\mathrm{XII}^{\mathrm{a}}$ dinastía egipcia ${ }^{11}$. Como podemos ver, estos procesos han acompañado al ser humano a lo largo de toda su existencia. A pesar de tan larga convivencia con esta patología se ha mantenido como una enfermedad incurable hasta prácticamente nuestros días. M.J. Sims en 1852 es el primero en publicar la curación de una FVV usando hilos de plata y por vía vaginal ${ }^{12}$. No obstante, fueron los trabajos de Von Dittil (1893) y sobretodo de Latzko (1942), los que permitieron avanzar de forma definitiva en la resolución de esta patología ${ }^{10}$.
Aunque sus causas etiológicas pueden ser diversas (Tabla III), sin lugar a dudas son las fístulas vesicovaginales yatrógenas las que representan el mayor contingente con el que nos vamos a tener que enfrentar. Cuando surge esta complicación nos encontramos con una mujer muy afectada, sobretodo psicológicamente, ya que se enfrenta a una complicación no esperada que le está generando un problema muy degradante para su persona, como es la incontinencia.

Las fistulas vesicouterinas, que representan aproximadamente el $10 \%$ restante, son de aparición mucho más reciente. Las primeras descripciones son del siglo XIX y se producían tras partos laboriosos e instrumentados. En la actualidad, la mayoría de ellas, son consecuencia de cesáreas, aunque también pueden producirse tras el uso de vacuum extractor, legrados o DIUs de larga permanencia (Tabla IV) ${ }^{13-15}$.

En el tratamiento de las fistulas vesicovaginales tendremos que diferenciar en primer lugar los procesos fistulosos yatrogénicos de los no yatrogénicos (neoplásicos, postrádicos, inflamatorios crónicos, cuerpos extraños, etc.) puesto que los tratamientos van a ser, en muchos casos, muy distintos. Hay que tener en cuenta una serie de consideraciones o medidas generales, después valorar la aplicación de un tratamiento conservador, en los casos que sea factible, como medida menos agresiva y finalmente si los anteriores no son eficaces o no son adecuados, deberemos plantear un tratamiento quirúrgico.

\section{TABLA III}

ETIOLOGÍA FÍSTULAS VESICOVAGINALES

\begin{tabular}{|c|c|}
\hline \multicolumn{2}{|c|}{ Congénita } \\
\hline \multicolumn{2}{|c|}{ Defecto en el desarrollo del seno urogenital. Muy raras } \\
\hline \multicolumn{2}{|c|}{ Adquirida } \\
\hline $\begin{array}{l}\text { Yatrogénica } \\
\text { (80\% países desarrollados) }\end{array}$ & No yatrogénica \\
\hline $\begin{array}{l}\text { Ginecología }(90 \%) \\
\text { (Histerectomía, colporrafia, biopsias) } \\
\text { Obstétrica }(9 \%) \\
\quad \text { (Cesárea, partos instrumentados) } \\
\text { Urológica }(0,5 \%) \\
\quad \text { (Cirugía I.U.E., cuello vesical, RTU vesical) } \\
\text { Digestiva }(0,5 \%) \\
\quad \text { (Cirugía colorrectal) }\end{array}$ & $\begin{array}{l}\text { Parto obstruido ( } 80 \% \text { en países no desarrollados) } \\
\text { Postradioterapia } \\
\text { Neoplasias pélvicas } \\
\text { Traumatismos: pélvicos, intravaginales } \\
\text { Enf. inflamatorias e infecciosas } \\
\text { Endometriosis, TBC, etc. } \\
\text { Cuerpo extraño: vaginal, vesical }\end{array}$ \\
\hline
\end{tabular}


TABLA IV

ETIOLOGÍA FÍSTULAS VESICOUTERINAS

\begin{tabular}{|l|l|}
\hline Obstétricas (95\%) & No obstétricas (5\%) \\
\hline Cesárea & Ginecológicas \\
\hline Parto distócico & (Cirugia vaginal, legrado, DIU) \\
\hline Uso de fórceps & Radioterapia pélvica \\
\hline Uso de Vacuum extractor & Neoplasias: uterina, vesical \\
\hline
\end{tabular}

Referente a las medidas generales a adoptar, debemos de tener en cuenta el uso de antibióticos para evitar las molestias inherentes a una infección urinaria, así como antifúngicos orales en el caso de infecciones vaginales por levaduras. También la aplicación de un tratamiento estrogénico (oral o transdérmico) en pacientes postmenopaúsicas o con menopausia inducida quirúrgicamente, que ayude a mejorar la calidad y elasticidad del epitelio tanto vaginal como vesical.

Dentro de los tratamientos conservadores, el primero a realizar será la colocación de una sonda vesical. Aunque hay datos dispares en la literatura, respecto a la eficacia de la misma, la tasa de éxitos se encuentra entre un 2 y un $24 \%$ en fístulas yatrógenas ${ }^{16-18}$. Nosotros hemos resuelto con esta maniobra el $20 \%$ de nuestras fístulas. Suelen tratarse de fístulas de pequeño tamaño y normalmente la resolución de la misma se produce en las primeras 4 semanas de permanencia con sonda vesical, por encima de este tiempo difícilmente se va a conseguir una curación. El mantenimiento prolongado de la sonda vesical en nuestros casos (media de 3 meses) se debió fundamentalmente a retrasos en la realización de pruebas diagnósticas y no a una intención terapéutica propiamente dicha. Otras opciones de tratamiento conservador descritas, con resultados dispares, son las oclusiones con fibrinógeno e incluso con tornillos metálicos ${ }^{19-21}$. También la fulguración endoscópica, que en fístulas pequeñas, se han publicado éxitos de un $56 \%$ y un $80 \%{ }^{22,23}$, aunque siempre existe el peligro de ampliar la fístula. En las fístulas vesicouterinas el tratamiento de sonda vesical asociado a hormonoterapia combinada (estrógenos + progestágenos), ha demostrado ser también de utilidad en aproximadamente un $5 \%$ de los $\operatorname{casos}^{24,25}$.
El tratamiento quirúrgico va a ser la solución definitiva en la mayoría de los casos. Existen múltiples técnicas quirúrgicas para el tratamiento de estás fístulas. Habiéndose descrito unos principios generales de la reparación quirúrgica que toda técnica debería cumplir. Básicamente son: identificación y tratamiento de los factores favorecedores (diabetes, arteriopatías, cuerpos extraños, infecciones, enfermedades inflamatorias, etc.); preparar los tejidos para que estén en óptimas condiciones; extirpar tejidos infectados y desvitalizados; aproximar tejidos limpios, bien vascularizados, sin tensión, en varias capas y con material reabsorbible; utilizar colgajos de interposición en casos complejos; y finalmente utilizar drenaje urinario postoperatorio continuo e ininterrumpido ${ }^{6}$.

Otros aspectos importantes a tener en cuenta con respecto al tratamiento quirúrgico, son el momento de la reparación y la vía de abordaje a utilizar. Clásicamente se hablaba de diferir la intervención entre 3 y 6 meses, con el objeto de que desaparezca la inflamación aguda y el edema, realizar el tratamiento adecuado de la infección y favorecer la regeneración vascular. Con estos principios se consigue la curación entre el 88 y el $100 \%$ de los $\operatorname{casos}^{10}$. Sin embargo, el hecho de que en la actualidad la etiología de las fístulas vesicovaginales sea diferente a la existente antaño, donde prevalecían las fístulas obstétricas, ha hecho plantear un tratamiento quirúrgico precoz. Diversos autores han publicado resultados similares a los previos realizando una cirugía precoz (antes de 3 meses), con la ventaja añadida que supone un menor daño psíquico y social para la paciente por la prontitud del tratamiento $26-29$. En nuestra serie, aunque solamente en 2 fístulas yatrógenas hemos realizado un tratamiento precoz, en ambos casos el resultado ha sido exitoso. En la actualidad se tiende a realizar el tratamiento quirúrgico lo antes posible, siempre que la fístula sea clara y limpia, retrasando la cirugía en las fístulas yatrógenas radiadas y las obstétricas grandes.

Han sido descritas múltiples técnicas quirúrgicas para la resolución de esta patología. Tradicionalmente se han venido dividiendo según su vía de abordaje en transabdominales y transvaginales, con o sin fistulectomía, así como, con o sin interposición de tejidos (Tabla V). Los resultados conseguidos por ambas vías son bastante 
TRATAMIENTO QUIRÚRGICO DE LAS FÍSTULAS VESICOVAGINALES

\begin{tabular}{|c|c|}
\hline \multicolumn{2}{|c|}{ Vía transabdominal } \\
\hline Extraperitoneal & Intraperitoneal \\
\hline $\begin{array}{l}\text { Fistulectomía: } \\
\text { - Fistulorrafia por planos } \\
\text { - Avanzamiento pared vesical posterior } \\
\text { (Gil-Vernet) } \\
\text { - Interposición de tejidos: peritoneo, duramadre } \\
\text { No fistulectomía: } \\
\text { - Injerto libre de mucosa vesical (Ostad) }\end{array}$ & $\begin{array}{l}\text { Bipartición vesical, fistulectomía y } \\
\text { fistulorrafia (O'Conor) } \\
\text { Asociado a interposición de tejidos: epiplón, } \\
\text { peritoneo, apéndices epiploicos, etc. }\end{array}$ \\
\hline \multicolumn{2}{|c|}{ Vía transvaginal } \\
\hline $\begin{array}{l}\text { Fistulectomía } \\
\text { Fistulorrafia por planos, avanzar mucosa } \\
\text { vaginal } \\
\text { Fistulorrafia e interposición de tejidos: } \\
\text { - Colgajo fibroadiposo labial pediculado } \\
\text { (Martius) } \\
\text { - Colgajo piel labial pediculada (Lehoczky) } \\
\text { - Colgajo peritoneal (Raz) } \\
\text { - Colgajo de músculo Gracilis (Hamlin) }\end{array}$ & $\begin{array}{l}\text { No fistulectomía } \\
\text { Incisión vaginal perifístula, cierre en varios } \\
\text { planos y avanzar mucosa vaginal o interponer } \\
\text { tejidos (Raz) }\end{array}$ \\
\hline
\end{tabular}

similares, estando todos ellos entre el $80 \mathrm{y}$ el $100 \%$ de éxitos ${ }^{17,18,30-36}$. Existe cierta controversia respecto a la vía más adecuada. Mientras para algunos autores el abordaje abdominal sigue siendo el más adecuado y lo consideran el patrón de referencia ${ }^{34}$, otros piensan que la vía vaginal es tan segura y efectiva como la abdominal, con menos morbilidad y menor estancia media hospitalaria ${ }^{29}$. Por tanto, la elección del abordaje va a depender mucho de las preferencias de cada cirujano. No obstante, existe acuerdo en que ciertos casos van a exigir siempre un abordaje abdominal, como son: acceso vaginal dificultoso, fístula muy próxima a orificios ureterales que precise un reimplante ureteral, fístula asociada a otra patología pélvica o abdominal (lesión ureteral, intestinal, etc.) y fístulas de gran tamaño, con abundantes esfacelos o irradiadas.

Las fístulas neoplásicas requerirán un tratamiento distinto. Estas exigirán un estudio individualizado de cada caso y una evaluación más detenida del tipo de tratamiento a realizar, según el grado de infiltración que sufra la vejiga. El abanico de posibilidades terapéuticas es amplio, llegando desde cirugías reconstructivas complejas con interposición de tejidos, hasta cirugías exeré- ticas y derivaciones urinarias o neovejigas ortotópicas. En otras ocasiones solamente será posible plantear tratamientos paliativos.

Las fistulas vesicouterinas van a precisar un abordaje transabdominal para su corrección sin que exista unanimidad respecto a la conveniencia de interponer tejidos, dado el escaso número de casos que aporta cada autor. Parece más adecuado interponer tejidos si persiste en la paciente deseo de nueva descendencia, para proteger la zona cicatricial de ambos órganos en los siguientes embarazos y partos ${ }^{14,37,38}$.

Para el tratamiento de las fístulas vesicovaginales, nosotros hemos sido partidarios de la vía transabdominal y de la técnica de $\mathrm{O}^{\prime}$ Conor con interposición de tejidos (peritoneo o epiplón) habiendo conseguido unos resultados acordes con los publicados por otros grupos (88,5\% de curación con la primera intervención), pero con un porcentaje de complicaciones postoperatorias no desdeñable, que afectaron al $33 \%$ de estas pacientes (3/9). Solamente en 3 fístulas de pequeña entidad nos decidimos por realizar fistulectomía y fistulorrafia en varios planos, con resolución de todas ellas y sin complicaciones. 
En la actualidad pensamos que el tratamiento de las fístulas vesicovaginales hay que particularizarlo en dependencia de su etiología y de la severidad de la misma. Existe un número elevado de fístulas yatrogénicas en las que una intervención por vía vaginal o abdominal extraperitoneal sin interponer tejidos las van a resolver con igual efectividad, menor morbilidad y recuperación más rápida. En estos momentos estamos interesados por incorporar la técnica de Gil-Vernet a la resolución de estas fístulas, dada su relativa sencillez y buenos resultados comunicados ${ }^{5,39}$. La interposición de tejidos puede reservarse para fístulas complejas y neoplásicas (Tabla VI), así como para las fístulas vesicouterinas. La revisión de la literatura y los resultados de nuestra serie nos han conducido a replantearnos nuestra estrategia terapéutica actual, tanto para las FVV como para FVU (Fig. 1).
TABLA VI

\section{FÍSTULAS COMPLEJAS}

- Fístulas gigantes: generalmente obstétricas (>5 cms)

- Fístulas asociadas a lesión uretral

- Múltiples

- Multioperadas: cicatrización severa

- Rádicas

- Radiadas

\section{CONCLUSIONES}

Las fístulas vesicogenitales generan un importante trastorno tanto físico como psíquico a las pacientes que las padecen.

El tratamiento conservador puede curar alrededor de un $20 \%$ de fístulas yatrógenas.

El tratamiento quirúrgico de las fístulas vesicovaginales yatrógenas simples y vesicouterinas

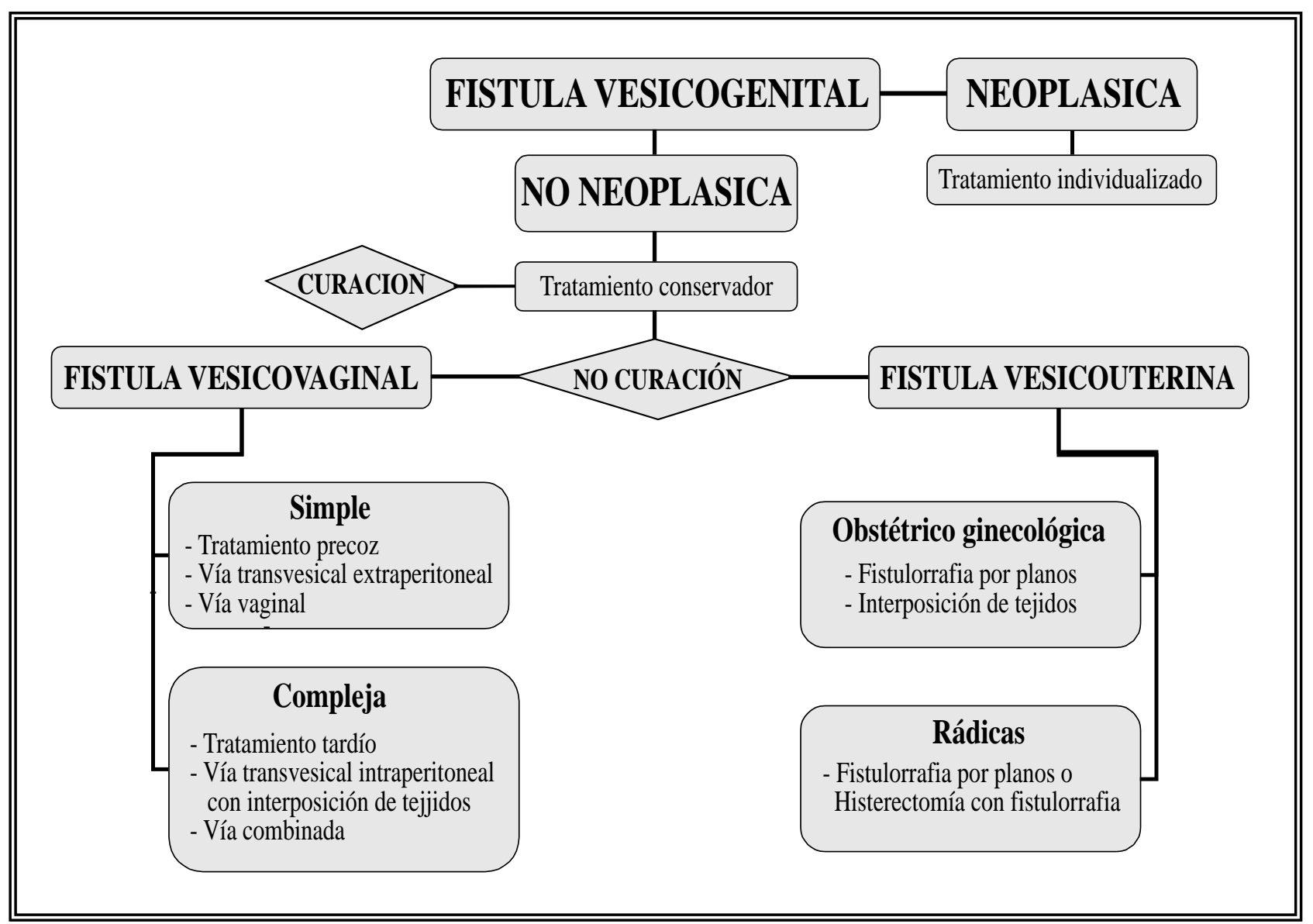


es eficaz en más del 90\% de los casos, independientemente de la técnica utilizada. Utilizar técnicas sencillas, que generen menor morbilidad, así como realizar cirugías precozmente no implica un peor resultado.

En las fístulas yatrógenas complejas es preferible utilizar colgajos de interposición (epiplon, peritoneo, etc), así como cirugías tardías, pues suelen ser donde con mayor frecuencia se producen los fracasos.

El tratamiento de las fístulas neoplásicas exige una evaluación exhaustiva de la paciente, particularizando en cada caso el tratamiento más adecuado.

\section{REFERENCIAS}

1. CARL P.: Vesico-ovarian fistula in suppurative ovarian inflamation and salpingitis. $J$ Urol 1990; 143: 352.

2. TURNER B.I.: Vesicosalpingovaginal fistula. Urology 1976; 8: 49.

3. Yazawa K.: Vesico-adnexal fistula following endometriosis of an ovary. Br J Urol 1997; 79 (4): 658.

4. ASANUMA H.: Congenital vesicovaginal fistula. Int $J$ Urol 2000; 7 (5): 195.

5. ARANGO TORO O.: Cierre autoplástico de las fístulas vesicovaginales con colgajo vesical posterosuperior (operación de Gil-Vernet). Actas Urol Esp 1997; 21: 604.

6. WOO H.H.: Tratamiento de la fístula vesicovaginal. Eur Urol (ed. española) 1996; 3: 163.

7. GOODWIN W.E.: Vesicovaginal and ureterovaginal fistulas: A sumary of 25 years of experience. J Urol 1980; 123: 370 .

8. FALANDRY L.: Vesicovaginal fistula in Africa. 230 cases. Presse Med 1992; 21: 241.

9. KELLY J.: Vesicovaginal and rectovaginal fistula. $J R$ Soc Med 1992; 85: 257.

10. MORENO SIERRA J.: Fístula vesicovaginal y otras fístulas genitourinarias infrecuentes. Clínicas Urológicas de la Complutense (Monografía fístulas del aparato genitourinario) 1998; 6: 339.

11. GÓMEZ VEGAS J.: Aspectos generales, clínica y diagnóstico de las fístulas ginecológicas. Bases terapéuticas. Clínicas Urológicas de la Complutense (Monografia fístulas del aparato genitourinario) 1998; 6: 313.

12. SIMS J.M.: On the treatment of vesicovaginal fistula. Am J Med Sci 1852; 23: 59.

13. JOZWIK M.: Actual incidence and cause of vesico-uterine fistula. Br J Urol 1998; 81: 341.

14. BENCHEKROUN A.: Vesicouterine fistulas. Report of 30 cases. Ann Urol (Paris) 1999; 33: 75.

15. YIP S.K.: Vesicouterine fistula: a rare complication of vacuum extraction in a patient with previous caesarean section. Br J Urol 1997; 80: 502.

16. DAVITS R.J.A.M.: Conservative treatment of vesicovaginal fistula by bladder drainage alone. Br J Urol 1991; 60: 155.

17. RAMOS C.: Fístulas vesicovaginales: corrección con duramadre liofilizada. Actas Urol Esp 1991; 15: 143.
18. TANCER M.L.: Observations on prevention and management of vesicovaginal fistula after total histerectomy. Surg Gynecol Obstet 1992; 175: 501.

19. PETERSON S.: Fibrin oclusion of a vesicovaginal fistula. Lancet 1979; 1: 933.

20. HEDELLIN H.: Fibrin oclusion of fistulas postoperatively. Surg Gynecol Obstet 1982; 154: 366.

21. AYCINEMA J.F.: Small vesicovaginal fistula. Urology 1977; 9: 543.

22. FALK H.C.: Nonsurgical closure of vesicovaginal fistulas. Obstet Gynecol 1957; 9: 538.

23. STOVSKY M.D., O־CONOR V.J.: Use de electrocoagulation in the treatment of vesicovaginal fistulas. J Urol 1994; 152: 1443 .

24. JOZWIK M.: Spontaneous closure of vesicouterine fistula. Account for effective hormonal treatment. Urol Int 1999; 62: 183.

25. HEMAL A.K.: Youssef's syndrome: an appraisal of hormonal treatment. Urol Int 1994; 52: 55.

26. PERSKY L.: Non-delay in vesicovaginal fistula repair. Urology 1979; 13: 273.

27. WANG Y.: Nondclayed transvaginal repair of high lying vesicovaginal fistula. $J$ Urol 1990; 144: 34.

28. BLANDY J.P.: Early repair of iatrogenic injury to the ureter or bladder after gynecological surgery. $J$ Urol 1991; 146: 761.

29. BLAIVAS J.C.: Early versus late repair of vesicovaginal fistulas: vaginal and abdominal approaches. J Urol 1995; 153: 1110.

30. DUPONT M.C., RAZ S.: Vaginal approach to vesicovaginal fistula repair. Urology 1996; 48: 7.

31. ISELIN C.E.: Transvaginal repair of vesicovaginal fistulas after hysterectomy by vaginal cuff excision. J Urol 1998; 160: 728.

32. OSTAD M.: Use of a free bladder mucosal graft for simple repair of vesicovaginal fistulae. Urology 1998; 52: 123.

33. CARR L.K., WEBSTER G.D.: Abdominal repair of vesicovaginal fistula. Urology 1996; 48: 10.

34. NESRALLAH L.J., GITTES R.F.: The O`Conor technique: The gold standard for supratrigonal vesicovaginal fistula repair. $J$ Urol 1999; 161: 566.

35. SÁNCHEZ MERINO, J.M.: Reparación transvesical de fístulas vesicovaginales no complicadas. Actas Urol Esp 2000; 24: 185 .

36. DÍAZ CALLEJA E.: Corrección quirúrgica de fístulas vesicovaginales mediante abordaje abdominal-transvesical. Comentarios a esta técnica y resultados a largo plazo. Arch Esp Urol 1997; 50: 55.

37. SOLER SOLER J.L.: Fístula vesicouterina: Aportación de dos nuevos casos. Actas Urol Esp 1998; 22: 146.

38. RIVAS DEL FRESNO M.: Fístula vesicouterina: a propósito de dos nuevos casos. Actas Urol Esp 1992; 16: 740.

39. GIL-VERNET J.M.: New surgical approach for treatment of complex vesicovaginal fistula. J Urol 1989; 141: 513.

Dr. F.J. Navarro Sebastián

Servicio de Urología

Hospital de Móstoles

C/ Rio Júcar, s/n

28935 Móstoles (Madrid)

(Trabajo recibido el 12 febrero de 2003) 